\title{
Neue Entwicklungen im Qualitätsmanagement
}

\author{
New perspectives for quality management in medicine
}

Die Messung medizinischer (Ergebnis-) Qualität ist unbestreitbar ein schwieriges Unterfangen. Ein Blick in die wissenschaftliche Literatur zeigt allerdings, dass trotz aller Bedenken ein weitgehender Konsens möglich ist über das, was bei der Behandlung einer bestimmten Krankheit oder Durchführung einer Operation die Ergebnisqualität im Wesentlichen ausmacht. Daraus folgt, dass die Definition und die Messung von Qualitätszielen auch unter den weniger strikt kontrollierten und kontrollierbaren Bedingungen der klinischen Praxis zwar schwierig, aber möglich sein wird.

Dass derartige Ansätze auch in der qualitativ bereits hoch entwickelten Medizin in den Industriestaaten notwendig und sinnvoll sind, hat die 1999 erschienene Metaanalyse „To Err is Human“ des Institute of Medicine gezeigt. Diese stellte anhand der Ergebnisse verschiedener Studien fest, dass hochgerechnet etwa mit 44000 bis 98000 Todesfällen in US-Krankenhäusern zu rechnen sei, die auf vermeidbare Behandlungsfehler zurückzuführen seien. Dadurch angestoßene sowie davon unabhängige Initiativen, beispielsweise die 100000 Lives Campaign des Institute for Healthcare Improvement in den USA, zeigen, dass Managementmaßnahmen zur Fehlerreduktion beitragen und die medizinischen Ergebnisse deutlich verbessern können. Gestützt wird diese Erfahrung durch Forschungsarbeiten, die einen erheblichen Einfluss organisatorischer Rahmenbedingungen - wie beispielsweise die strikte Einhaltung von Leitlinien - auf die Ergebnisqualität belegen.

\section{Qualitätsverbesserung als ärztlicher Auftrag und Managementaufgabe $\checkmark$}

Diese Erkenntnisse und der zunehmende Wettbewerb der Leistungserbringer führen dazu, dass Maßnahmen zur Qualitätsverbesserung zunehmend nicht nur die Ärzteschaft betreffen, die traditionell in diesem Bereich engagiert ist, sondern auch eine Herausforderung für das Management der Kliniken sowie für die Gestaltung der Rahmenbedingungen durch Krankenkassen und Politik darstellen. Diese im Prinzip zu begrüßende Verbreiterung der Basis wird in der Medizin gelegentlich als „Einmischung von außen“ empfunden und daher nicht immer freudig begrüßt. Die Ergänzung der innerärztlichen Verfahren um ein konstruktives Zusammenwirken von Medizin und Management in der Steuerung und Beeinflussung von Qualität ist aber gleichwohl mittelfristig notwendig und sinnvoll.
Unterstützt wird diese Entwicklung durch die neuen Möglichkeiten, die sich aus der Verfügbarkeit zunehmend detaillierter medizinischer Informationen insbesondere in den stationären Abrechnungssystemen und damit in den Routinedaten (den sogenannten „administrative data“) der Krankenhäuser und Krankenkassen ergeben. Dies gilt aufgrund der verbreiteten Einführung leistungsbezogener Abrechnungsverfahren (Stichwort DRG) und erheblich verbesserter EDV-technischer Auswertungsmöglichkeiten international. Die in diesem Abstractband veröffentlichten Beiträge zum Kongress „Qualitätsmessung und Qualitätsmanagement mit Routinedaten“ geben einen Überblick über den Stand dieser neuen Ansätze und zeigen neben den Erfahrungen mit der Methode insbesondere die Möglichkeiten auf, die sich aus der zeitnahen und umfassenden Verfügbarkeit von Qualitätsindikatoren ergeben, die bereits in den Kliniken ohne Zusatzaufwand aus vorhandenen Daten abgeleitet werden können. Darüber hinaus beinhaltet vor allem die hier vorgestellte, völlig neue Methodik zur routinemäßigen Ableitung von Langzeitergebnissen aus den ohnehin vorhandenen Daten der Krankenkassen, deren Ermittlung mit den klassischen Erhebungsverfahren wegen des enormen Aufwandes allenfalls in umschriebenen Forschungsprojekten möglich war, erhebliche Chancen für die Weiterentwicklung der Qualitätsmessung und damit auch des Qualitätsmanagements.

\section{Qualitåt als Vergůtungselement}

Die Möglichkeit, Qualitätsmerkmale beidseits nämlich auf Seiten der Leistungserbringer und der Krankenkassen - zu messen, ist ein wesentlicher Schritt hin zur Überprüfbarkeit und Objektivierbarkeit und macht Qualität prinzipiell verhandelbar. Dies beeinflusst nicht zuletzt auch die Entwicklung ergebnisorientierter Vergütungssysteme in der Medizin, die damit aus dem Bereich der ökonomischen Theorie in die Praxis übergehen. Auch diese Thematik ist daher ebenso wie die Frage der möglichen Berücksichtigung von Qualitätsaspekten in der Krankenhausplanung Gegenstand verschiedener Beiträge in diesem Heft.

Das neue DRG-System von Medicare in den USA beinhaltet eine erste systemweite Umsetzung solcher Ansätze und geht damit über andere bereits bekannte, teils sehr umschriebene bzw. spezifische Projekte hinaus (siehe T. Valuck, S. S140). Das Problem der statistischen Unsicherheit von Qualitätskennzahlen, welches eine darauf basie-

\begin{abstract}
T. Mansky ${ }^{1}$
M. Heberer ${ }^{2}$

J. Klauber $^{3}$
\end{abstract}

Qualitätsmanagement

Schlüsselwörter

Qualitätsindikatoren

Qualitätsmanagement

$\checkmark$ Routinedaten

Key words

$\checkmark$ quality indicators

quality management

administrative data

Institut

${ }^{1}$ HELIOS Kliniken

2Universitätsspital Basel

3 Wissenschaftliches Institut der AOK

Bibliografie

DOI 10.1055/s-0028-1085581

Dtsch Med Wochenschr 2008;

133: S133-S134 - (c) Georg Thieme Verlag KG Stuttgart · New York · ISSN 0012-0472

Korrespondenz PD Dr. Thomas Mansky Abteilungsleiter Medizinische Entwicklung HELIOS Kliniken GmbH Friedrichstr. 136 10117 Berlin Tel. +49 (30) 521 321-140 eMail thomas.mansky@ helios-kliniken.de 
rende Vergütung erschwert, wird dadurch umgangen, dass in standardisierter Form auf Einzelfälle Bezug genommen wird. Bestimmte vermeidbare Komplikationen, die bisher zu einer Schweregraderhöhung im Einzelfall und damit zu einer höheren Vergütung geführt hätten, werden im neuen DRG-System von Medicare nicht mehr als vergütungsrelevant berücksichtigt, d.h. komplikationsfreie Verläufe werden dadurch aufgewertet. Sowohl hinsichtlich der praktischen Umsetzbarkeit als auch hinsichtlich der Anreizwirkungen ist dies ein hochinteressanter Ansatz in der Umsetzung einer ergebnisorientierten Vergütung. Auch hier zeigt sich, dass die weitere Entwicklung auf jeden Fall eine engere, konstruktive Zusammenarbeit von Medizin und Management in Qualitätsfragen erforderlich machen wird.

\section{Qualität als zweite Dimension im Gesundheitssystemvergleich}

Die Verfügbarkeit besserer, objektivierbarer Informationen zur Ergebnisqualität eröffnet auch auf Systemebene neue Möglichkeiten. Die Diskussion der Leis- tungsfähigkeit von Gesundheitssystemen erfolgt bisher überwiegend kostenorientiert. Der Beitrag der OECD zeigt beispielhaft, dass das Bestreben dahin geht, die Kostenvergleiche um ein auf Indikatoren beruhendes Qualitätsbenchmarking zu erweitern (siehe N. Klazinga, S. S145). Es handelt sich beim Vergleich nationaler Gesundheitssysteme, wie er von der OECD vorgenommen wird, zwar um eine andere, abstraktere Betrachtungsebene als beim Vergleich einzelner Leistungserbringer. Vor allem der Detaillierungsgrad wird auf der Ebene der Leistungserbringer höher sein als bei der OECD. Dennoch gibt es bei einigen der eingesetzten Indikatoren erwartungsgemäß Überschneidungen (beispielsweise Sterblichkeit bei Herzinfarkten). Insofern gibt es durchaus Wechselwirkungen der verschiedenen Qualitätsindikatorenprojekte untereinander.

\section{Qualität als Herausforderung für die Systemgestaltung}

Die Verbesserung der ergebnisorientierten Qualitätsmessung, die darauf basierenden Veränderungen im Qualitätsma- nagement und die Bestrebungen, ergebnisorientierte Vergütungselemente einzuführen, stellen konvergierende Entwicklungsstränge dar. Die Tagung und dieses Supplement geben beispielhaft den aktuellen Entwicklungsstand wieder und zeigen die Entwicklungsdynamik in diesem Bereich. Auch bei gleichen Kosten kann im Gesundheitssystem ebenso wie in anderen Wirtschaftsbereichen die Qualität der erbrachten Leistungen durchaus unterschiedlich sein.

Eine Herausforderung für alle an der Systemgestaltung Beteiligten wird es daher sein, über Ergebnistransparenz, die sinnvolle Verknüpfung ökonomischer Anreize und medizinischer Zielsetzungen und andere Maßnahmen Rahmenbedingungen zu schaffen, die trotz des unvermeidlich begrenzten Finanzierungsrahmens im Gesundheitswesen die Leistungserbringer in ihrem Bemühen um die weitere Verbesserung der Ergebisqualität möglichst wirkungsvoll unterstützen. Ziel ist es ferner, die überwiegende Kostendiskussion im Gesundheitswesen um eine objektivierbare Qualitätsdiskussion zu ergänzen, um so zu einer echten Kosten-/ Nutzen-Bewertung zu gelangen.
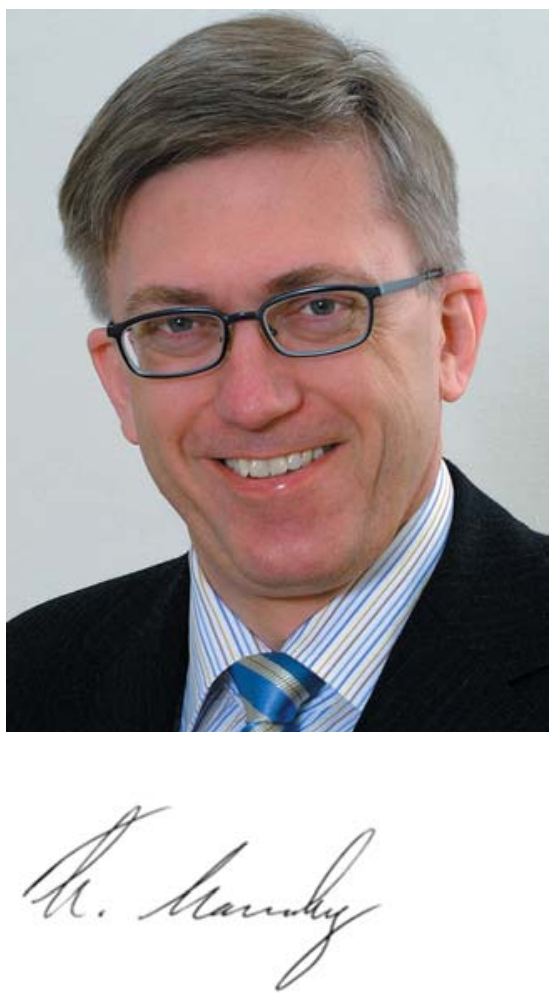

PD Dr. Thomas Mansky HELIOS Kliniken
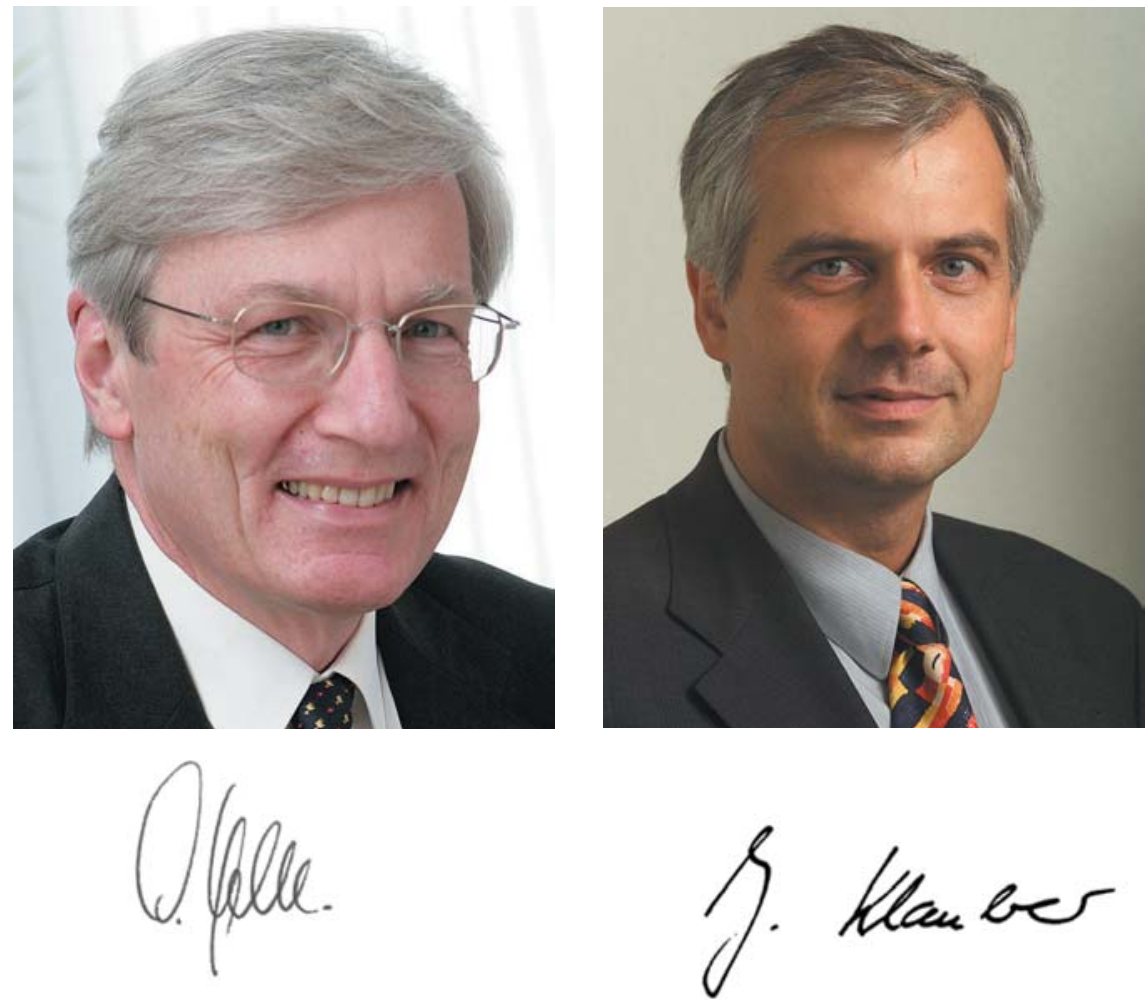

Prof. Dr. Michael Heberer

Universitätsspital Basel 